\title{
English Pronunciation Errors: A Case Study of Amhara and Oromia Regions of Ethiopia
}

Ojo, B. J (PhD) \& Umera-Okeke, Nneka

\begin{abstract}
:
The main goal of this study is oral fluency, that is, the ability to express oneself intelligibly, reasonably, accurately and without too much hesitation without which communication breaks down. In pursuit of this intelligibility between speaker and hearer, the researchers observed through formal and informal settings to find out the deficiencies in the speeches of speakers from the two regions under study that calls for immediate attention. Efforts were made to highlight the pronunciation errors, give possible reasons why they may be occurring and provided plausible solutions.
\end{abstract}

\section{Introduction}

The English Language is used as a foreign language in Ethiopia. Even at that, the teaching of English in schools does not start until grade seven of the education. The language of instruction and communication at all levels is the respective regional language -Amharic in Amhara region and Afan Oromo in Oromia region. Government businesses are mostly conducted in the regional languages. It is observed that circulars are usually written in the native language in the universities. Though stated that the language of instruction from higher grade to the university level is English, most indigenous lecturers still deliver their lectures in their native language. This is because some are not proficient in the English Language having gone through the same system, and the students who all through their lives have been using their native languages are unable to understand lectures delivered in English. This is 
the shock of most expatriates in Ethiopia who run into the problem of being understood by these students.

Some of the students and teachers who can speak English render some pronunciations inaccurately. This could be attributed to the earlier teachers of English in Ethiopia and the influence of the sound system of the native language which they use for everything. This is possible because a learner may encounter sounds in English that are not part of the sound inventory of the learner's native language. Thirdly, difficulties may also arise because the rules for combing sounds into words are different in the learners' native language. As a result of these difficulties, the native language affects not just the ability of the learners to produce English sounds, but also their ability to hear the sounds.

These assumptions were confirmed by Avery and Ehrlich (1997) when they identified the factors that contribute to the inability of the second language learners (also foreign language learners) to acquire the sound system of a second language. The factors according to them include biological, socio-cultural, personality and linguistic factors.

\section{Biological Factors:}

Avery and Ehrlich (1997) used the "critical period hypothesis" to explain this factor. This hypothesis holds that languages are learned differently by children and adults. While child second language learner almost always attains native-like pronunciation, the adult learners almost always have foreign accent. Most speakers of English in Amhara and Oromia regions have this foreign accent because English is not learnt early enough in their lives.

\section{Socio-Cultural Factors:}

Socio-cultural factors play an important role in Ethiopia. The more a learner identifies and affiliates with his language and culture, the more he loses grip of the sound system of 
English. Conversely, early training in the target language and its pronunciations brings about improvement in the learners.

\section{Personality Factor:}

The personality of the learners should not be left out. Extroverts who are out-going with the wish to socialize learn to speak better than introverts who are always shy to interact with others.

\section{Historical Factor:}

Historically, the problem of acquisition and usage of the English language in Ethiopia can be traced partly to the takeover of education by the natives after the fall of the Imperial regime. After the liberation of the country in 1942, the British as partners in the liberation struggle asserted their influence on the organization of educational system in the country (Seyoun, 1996). As would be expected, the language of instruction as the English language. But with the death of Imperial regime and the taken-over of the Dergue regime in 1974, the foreign teachers (Americans, Britons, and the Indians) left. This led to a dire shortage of qualified teachers both at the primary and secondary school levels. Before the exist of the foreign teachers, the observation was that the proficiency of the most native teachers and students in English was anything but poor (Seyoun, 1996). In fact, the language was seen as a medium of obstruction rather than of instruction. Therefore, the exist of the foreign teachers, many untrained teachers were recruited to teach in the schools with their little knowledge of English. This led to the agitation for teaching in Amharic language since the teachers were not proficient in the English language.

Again, the disappearance of the few educated Ethiopians either to war or dispersion by 1974 dealt a serious blow to the use of English as a medium of instruction. Many of these educated few found themselves in Europe and America and after their studies decided not to come home; thus educating was left largely in the hands of untrained teachers. 
Coupled with this was the policy of secrecy on educational matters of the regime at that time and the strengthening of Amharic as the medium of instruction up till recently when English language was introduced as a medium of instruction at the higher level of education in Ethiopia. It is not uncommon to see the native English language teachers using Amharic to teach the English language and other subjects like Mathematics and Physics during classes.

The idea of teaching students to write, and not to speak, is another very vital set back to the English Language acquisition in Ethiopia.

\section{Linguistic Factors:}

The learners' native language to a large extent determines the nature of his foreign accent. The sound patterns of the native languages may be transferred into the second language. the pronunciation errors that second/foreign learners made were not just random attempt to produce some familiar sounds but they rather reflect the sound inventory, rules of combination, stress and intonation patterns of the native language.

It is the combination of these factors that play a role in the errors noticed in English pronunciations in the regions studied.

\section{Statement of Problem:}

It has been noticed that both students and the elite members of Amhara and Oromia regions of Ethiopia make the following production errors in the use of the English Language:

- Inserting the sound /I/ before the plural morpheme /s/

- Generalizing all the past tense morphemes to /ed/

- Insistence on the pronunciation of the liquid sound $/ r /$ anywhere it occurs in a word. 
- Pronunciation of the voiced velar plosive $/ \mathrm{g} /$ in word final position even when the realization should be the velar nasal / $\mathrm{g} /$

- The pronunciation of the dental sounds / $\theta$, $\partial /$ as voiced alveolar fricative /z/

- Inability to note the silent letters in English

\section{Methodology:}

The researchers made use of both formal and informal methods in collecting the data for this study. Formally, two universities were used - Haramaya University in Oromia region and Bahir Dar university in Amhara region. In the course of classroom teaching, students were given passages to read. The errors perceived by the researchers were documented in a written form. More were also recorded while the students interacted with the researchers-teachers. The researchers recorded the re-occurrence of most errors observed in the classroom.

There were also informal documentations of errors. It is informal because the native speakers never knew they were being observed. This has to do with every day conversations and interaction with colleagues and members of the public. Two important workshops/seminars were attended by the researchers at both universities under study. They are workshop on the review of post graduate curriculum for College of Agriculture at Haramaya University and $25^{\text {th }}$ Annual Seminar of the faculty of Education in Bahir Dar University, Ethiopia. Each lasted for two days. Papers were presented by highly educated members of the university communities. Discussions:

This section of the study looked into the pronunciation errors as well as stated what they ought to be. Some of these errors noticed were as follows: 
1. Inserting the sound /I/ before the regular plural morpheme /s/

Most words that end in /s/ were not properly pronounced. The sound /I/ was always imposed after that consonant as if the intention is to maintain a CVC structure. We heard erroneous pronunciations like:

\begin{tabular}{|c|c|c|}
\hline Words & $\begin{array}{l}\text { Erroneous } \\
\text { Pronunciation }\end{array}$ & $\begin{array}{l}\text { Correct } \\
\text { Pronunciation }\end{array}$ \\
\hline Student & */stju:dentIs/ & /stju:dnts/ \\
\hline Aspects & */æspektIs/ & /æspekts/ \\
\hline Fields & */fi:Idis/ & /fi:Ids/ \\
\hline Involves & */Invblves/ & /InvDlves/ \\
\hline Pests & */pestIs/ & /pests/ \\
\hline Reports & */ripo:tis/ & /rips:ts/ \\
\hline Agents & * /eIdzəntIs/ & /eIdzonts/ \\
\hline Supermarke & et*/su:pərma:ketIs/ & /su:pərma:kets/ \\
\hline Slides & */slaidis/ & /slaids/ \\
\hline Contents & */knotentis/ & /kbntents/ \\
\hline Length & */lengtIs/ & /len's's/ \\
\hline Pupils & */pju:pIlis/ & /pju:pls/ \\
\hline Respondent & ts*/respondəntIs/ & /respondənts/ \\
\hline Comments & */kbmentIs/ & /kbments/ \\
\hline Instruments & ts*/instrəməntis/ & /instrəmənts/ \\
\hline Scientists & */saiəntistis/ & /saiəntists/ \\
\hline Points & $* /$ poıntIs/ & /pounts/ \\
\hline Chemists & */kemIstis/ & /kemists/ \\
\hline
\end{tabular}

The regular English plural is formed by the addition of s/es to the base word. They are realized in three different forms governed by phonological conditions:

i. It is realized as $/ \mathrm{s} /$ after voiceless sounds except the hissing sounds $-/ \mathrm{s}, \int, \mathrm{t} \int / \mathrm{as}$ in: 


\begin{tabular}{|c|c|}
\hline $\begin{array}{l}\text { Cats /kæts/ } \\
\text { Sits /sits/ } \\
\text { Myths /mi } \theta \text { s/ }\end{array}$ & $\begin{array}{l}\text { books /buks/ } \\
\text { goats /gəひts/ } \\
\text { stops /stpps/ }\end{array}$ \\
\hline
\end{tabular}

ii. /z/ occurs after voiced sounds, that is, all vowels ad voiced consonants except hissing sounds as in :

Dogs /dngz/ bags /bægz/ leaves / li:vz/

Girls /ga:lz/ boys /bolz / sings /sinz/

Mills /milz/ cans /kænz/ pegs /pegz/

iii. /iz/ occurs after the hissing sounds (sibilants) /s, z, 3, $\mathrm{d} 3, \mathrm{t} / \mathrm{as}$ in:

Houses /hauziz/ beaches /br:t IIz/ badges /bæ dz Iz/

Churches / t $\int 3: t \int \mathrm{Iz} /$ dishes / $\mathrm{d}_{\mathrm{I}} \int \mathrm{Iz} /$ ridges/ridz Iz/

Messages/mesidz Iz/ buses /b^siz/

benches /bent $\int \mathrm{Iz} /$

2. The next area of error was the generalization of the entire regular past tense morpheme to /ed/. This can be exemplified in the pronunciation of the following words which were observed and recorded.

\begin{tabular}{|c|c|}
\hline Words & $\begin{array}{l}\text { Erroneous } \\
\text { Pronunciation }\end{array}$ \\
\hline Asked & */a:sked/ \\
\hline Advanced & */ədva:nsed/ \\
\hline Involved & */Involved/ \\
\hline Convinced & */kənvinsed/ \\
\hline Worked & */w3:ked/ \\
\hline Fixed & $* /$ fIksed/ \\
\hline Changed & */tكeindzed/ \\
\hline Concerned & */kəns3:ned/ \\
\hline Advised & */ədvaIsed/ \\
\hline
\end{tabular}

Correct

Pronunciation

/a:skt/

/ədva:nst/

/Inolvd/

/kənvinst/

/w3:kt/

/fikst/

/t $\int e I n d z d /$

/kənsz:nd/

/ədvaIzt/ 


\begin{tabular}{|c|c|c|}
\hline $\begin{array}{l}\text { Depressed } \\
\text { Designed } \\
\text { Described } \\
\text { Assumed } \\
\text { Launched }\end{array}$ & 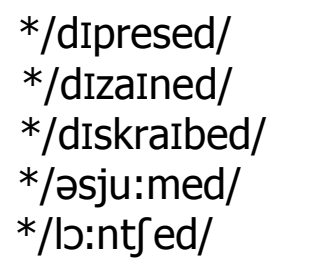 & $\begin{array}{l}\text { /diprest/ } \\
\text { /dizaind/ } \\
\text { /diskraibd/ } \\
\text { /əsju:md/ } \\
\text { /lo:nttt/ }\end{array}$ \\
\hline $\begin{array}{l}\text { Converged } \\
\text { Contrived } \\
\text { Forced } \\
\text { Collapsed }\end{array}$ & $\begin{array}{l}\text { */kənv3:dzed/ } \\
\text { */kəntraIved/ } \\
\text { */fo:ksed/ } \\
\text { */kəlæpsed/ }\end{array}$ & $\begin{array}{l}\text { /kənv3:dsd/ } \\
\text { /kəntraivd/ } \\
\text { /fo:st/ } \\
\text { /kəlæpst/ }\end{array}$ \\
\hline Repulsed & */rip ^lsed/ & /rip^lst/ \\
\hline $\begin{array}{l}\text { Arranged } \\
\text { Performed }\end{array}$ & $\begin{array}{l}\text { */əreIndzed/ } \\
\text { */pəfo:med/ }\end{array}$ & $\begin{array}{l}\text { /əreIndzd/ } \\
\text { /pəfo:md/ }\end{array}$ \\
\hline
\end{tabular}

There is this insistence on pronouncing the -ed wherever it occurs. Just like the plural morpheme, we have three different realizations of the regular past tense morpheme. The sound /ed/ is not one of them. They are realized as $/ d, t$, id/ under the following phonological conditions:

i. /t/ or /d/ at the end of a word plus -ed is realized as /Id/

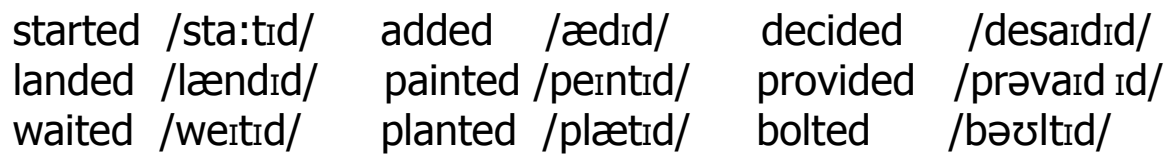

ii. The voiceless sounds $/ p, k, f, s, \int, t /$ plus -ed is realized as /t/:

\begin{tabular}{|c|c|c|c|c|}
\hline $\begin{array}{l}\text { dreamt } \\
\text { roped } \\
\text { talked } \\
\text { missed }\end{array}$ & $\begin{array}{l}\text { /dremt/ } \\
\text { /rəorpt/ } \\
\text { /to:kt/ } \\
\text { /mist/ }\end{array}$ & $\begin{array}{l}\text { knocked } \\
\text { walked } \\
\text { smashed } \\
\text { asked }\end{array}$ & $\begin{array}{l}\text { /nokt/ } \\
\text { /wo:kt/ } \\
\text { /smæSt/ } \\
\text { /æskt/ }\end{array}$ & $\begin{array}{l}\text { clapped /klæpt/ } \\
\text { coughed /knft/ } \\
\text { fixed /fikst/ } \\
\text { faced /feist/ }\end{array}$ \\
\hline
\end{tabular}


iii. All other voiced sounds except /d/ plus -ed is realized as /d/

\begin{tabular}{|c|c|c|c|}
\hline Stoned & /stərnd/ & solved /s^lvd/ & hurried /h^rid/ \\
\hline $\begin{array}{l}\text { Played } \\
\text { Sued } \\
\text { Bowed }\end{array}$ & $\begin{array}{l}\text { /pleid/ } \\
\text { /sju:d/ } \\
\text { /baud/ }\end{array}$ & $\begin{array}{l}\text { cuddled /k^dld/ } \\
\text { begged /begd/ } \\
\text { tagged / tægd/ }\end{array}$ & $\begin{array}{l}\text { praised /preiz } \\
\text { bribed /braibd } \\
\text { lived/livd/ }\end{array}$ \\
\hline
\end{tabular}

3. Another observable area of erroneous pronunciation was the insistence on the pronunciation of the liquid $/ r /$ no matter where it occurs. Some of the words erroneously pronounced were:

\begin{tabular}{|c|c|c|}
\hline Words & $\begin{array}{l}\text { Erroneous } \\
\text { Pronunciation }\end{array}$ & $\begin{array}{c}\text { Correct } \\
\text { Pronunciation }\end{array}$ \\
\hline Car & */ka:r/ & /ka: (r)/ \\
\hline Desert & */dezərt/ & /dezət/ (n), /dizs:t/ (v) \\
\hline Iron & */aIrDn/ & /aIən/ \\
\hline Carp & */ka:rp/ & /ka:p/ \\
\hline Card & */ka:rd/ & /ka:d/ \\
\hline Teacher & 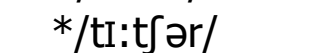 & 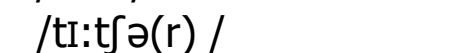 \\
\hline Girls & */g3:rlis/ & /g3:ls/ \\
\hline Third & 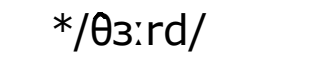 & /өз:d/ \\
\hline $\begin{array}{l}\text { Thirst } \\
\text { First } \\
\text { Counter } \\
\text { Court } \\
\text { ort }\end{array}$ & $\begin{array}{l}\text { */O3:rst/ } \\
\text { */f3:rst/ } \\
\text { */kauntər/ } \\
\text { */ko:rt/ } \\
\text { */so:rt/ }\end{array}$ & $\begin{array}{l}\text { /O3:st/ } \\
\text { /fs:st/ } \\
\text { /kauntə(r)/ } \\
\text { /ko:t/ } \\
\text { /so:t/ }\end{array}$ \\
\hline $\begin{array}{l}\text { ugar } \\
\text { ister } \\
\text { octor } \\
\text { etter }\end{array}$ & $\begin{array}{l}\text { */sugar/ } \\
\text { */sIstər/ } \\
\text { */dnktər/ } \\
\text { */betər/ }\end{array}$ & $\begin{array}{l}\text { /sugə(r)/ } \\
\text { /sistə(r)/ } \\
\text { /dpktə(r)/ } \\
\text { /betə(r)/ }\end{array}$ \\
\hline $\begin{array}{l}\text { lother } \\
\text { ransport }\end{array}$ & $\begin{array}{l}\text { */m^ðər/ } \\
\text { */trænspo:rt/ }\end{array}$ & $\begin{array}{l}\text { /m^ðə(r)/ } \\
\text { /trænspว:t/ }\end{array}$ \\
\hline
\end{tabular}


Transfer

Escort

Export

Heart */trænsfər/

*/isko:rt/

*/Ikspo:rt/

*/ha:rt/ /trænsfə(r)/

/isko:t/

/Ikspo:t/

/ha:t/

The liquid sound $/ r /$ is easily pronounced when it occurs immediately before vowels as in rate, rat, race, etc. When words are said in isolation, $/ r$ / occurs only in initial and medial positions as in:

Initial Position
Rat
Race
Run
Rock
Rag
Rope
Read

$$
\begin{aligned}
& \text { Medial Position } \\
& \text { pray, around } \\
& \text { mirror, barrow } \\
& \text { sorry, bright } \\
& \text { fury, tray } \\
& \text { spirit, pirate } \\
& \text { very, during } \\
& \text { from, carry }
\end{aligned}
$$

This is the reason why it is silent in word final positions as in car $/ \mathrm{ka}: / . / \mathrm{r} /$ is silent before consonants and final positions unless the word is followed by another word beginning with a vowel. For example:

\section{In Isolation:}

Art /a:t/

Curl /k3:l/

Heart /ha:t/

Car /ka:t/

My father /maI fa:ðə/

There with / ðeə wIð/

When followed by other word beginning with a vowel, it is pronounced out as in: 
A car arrived /ə ka:r əraIvd/

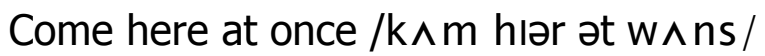

There upon /ðeər əppn/

There under /ðeər $\wedge$ ndə(r)/

4. The next problematic area was the pronunciation of the voiced velar plosive $/ \mathrm{g} /$ in word final position even when the realisation should be the velar nasal / $\mathrm{g} /$. Examples were:

Words

\begin{tabular}{|c|c|}
\hline $\begin{array}{l}\text { Gang } \\
\text { Hang } \\
\text { Robbing }\end{array}$ & $\begin{array}{l}\text { */gæng/ } \\
\text { */hæng/ } \\
\text { */robing/ }\end{array}$ \\
\hline Thing & $* / \theta$ Ing / \\
\hline $\begin{array}{l}\text { Coming } \\
\text { Morning } \\
\text { Sing } \\
\text { Regarding }\end{array}$ & $\begin{array}{l}\text { */k^mIng/ } \\
\text { */mo:nIng/ } \\
\text { */sing/ } \\
\text { */riga:ding/ }\end{array}$ \\
\hline $\begin{array}{l}\text { Running } \\
\text { Going } \\
\text { Refreshing } \\
\text { Sang }\end{array}$ & 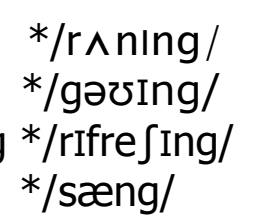 \\
\hline $\begin{array}{l}\text { Sung } \\
\text { Whoppin }\end{array}$ & $\begin{array}{l}\text { */s^ng/ } \\
\text { */wDpIng/ }\end{array}$ \\
\hline
\end{tabular}

\section{Erroneous}

Correct

Pronunciation

Pronunciation

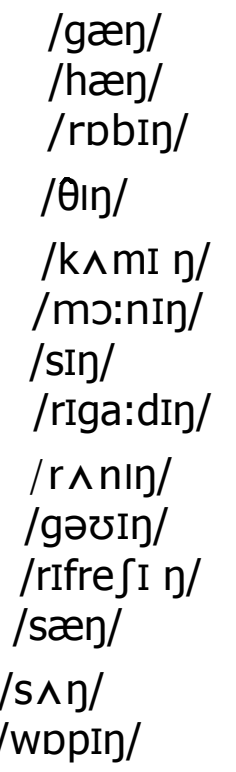

A lot of words ending in $\mathrm{ng}$ are generally realized as $/ \mathrm{m} /$. The soung $/ \mathrm{g} /$ is pronounced in word initial, medial and final positions as in gun, glass, eager, hunger, dug, mug and so on. Apart from being realized as $/ \mathrm{y} /$ in $\mathrm{ng}$ spellings, it is silent when followed by $\mathrm{m}$ or $\mathrm{n}$ in the same syllable as in gnat, gnaw, phlegm, sign, feign, etc. 
5. The pronunciation of inter-dental sounds / $\theta, \partial /$ as voiced alveolar fricative /z/:

The sounds $/ \theta /$ and $/ \partial /$ are referred to as inter-dental sounds because the tongue is placed between the teeth in its production. The two th sounds are notoriously difficult for foreign/second language learners because it does not exist in a lot of indigenous languages. Ethiopians realize th as / $z /$. You hear such erroneous constructions as:

\begin{tabular}{|c|c|c|}
\hline $\begin{array}{l}\text { Word } \\
\text { Then } \\
\text { That } \\
\text { The }\end{array}$ & $\begin{array}{l}\text { Erroneous } \\
* / \text { zen/ } \\
* / \text { zæt } \\
* / \text { zə/ }\end{array}$ & $\begin{array}{l}\text { Right } \\
\text { /ðеn/ } \\
\text { /ðæt/ } \\
\text { /ðə/ or /ðI/ }\end{array}$ \\
\hline $\begin{array}{l}\text { The other } \\
\text { Whether } \\
\text { This } \\
\text { Those } \\
\text { Father }\end{array}$ & $\begin{array}{l}\text { */zI ว:zə/ } \\
\text { */wezər/ } \\
\text { */zIs/ } \\
\text { * /zəひz/ } \\
\text { */fa:zər/ }\end{array}$ & $\begin{array}{l}\text { /ðІ ^ðə(r) / } \\
\text { /weðə(r) / } \\
\text { /ðIs/ } \\
\text { /ðəひz/ } \\
\text { /fa: ðə(r) / }\end{array}$ \\
\hline $\begin{array}{l}\text { Mother } \\
\text { Thought }\end{array}$ & $\begin{array}{l}* / \mathrm{m} \wedge z \partial r / \\
* / \mathrm{zo:t/}\end{array}$ & $\begin{array}{l}\text { /m^ðə(r) / } \\
/ \theta \supset: t /\end{array}$ \\
\hline
\end{tabular}

These sounds should be differentiated for the foreign language learners. While / $\theta /$ and / $\partial /$ are inter-dental fricatives - the first voiceless and the latter voiced, the sound/z/ is a voiced alveolar fricative. The alveolar sounds are produced with the tip of the tongue touching the alveolar ridge. $/ \partial /$ is found in words like the, this, that, these, those, then, than, there, though, them, mother, father, brother, etc. / $\theta$ / is found in think, thank, thought, mouth, cloth, path, etc.

6. Finally is the speakers' inability to note the existence of a lot of silent letters in English. As for them, every letter in a word must be pronounced. Below is a collection of some 
English letters and how they are to be pronounced as compiled by Umera-Okeke (Unpublished).

\section{Silent B}

When the letter $b$ comes after $m$, or when it comes before $t$ in words, the $b$ is silent in such words:

\begin{tabular}{|c|c|c|}
\hline 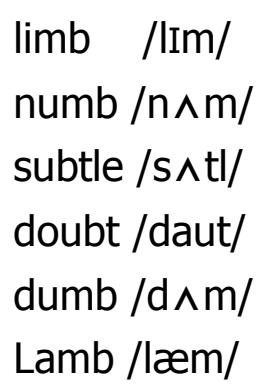 & $\begin{array}{l}\text { thumb / } \theta \wedge \mathrm{m} / \\
\text { womb /wu:m/ } \\
\text { debtor /detə/ } \\
\text { Plumb /pl^m/ } \\
\text { crumb /kr^m/ } \\
\text { succumb/sək^m/ }\end{array}$ & $\begin{array}{l}\text { comb /kəəm/ } \\
\text { debt /det/ } \\
\text { doubtful /dautfəl/ } \\
\text { climb /klaIm/ } \\
\text { tomb/tu:m/ } \\
\text { bomb /bom/ }\end{array}$ \\
\hline
\end{tabular}

\section{Silent C}

Muscle /m^sl/ Scene /si:n/ abscond / əbskbnd/

Science /saIəns/ Abscess /æbses/ indict/IndaIt/

Scepter /septə/ victuals /vitlz/

\section{Silent D}

width / wi $\theta$ /

handkerchief /hænkət]If/

sandpaper/sænpeIpə/

\section{Silent G}

\begin{tabular}{|c|c|c|}
\hline $\begin{array}{l}\text { gnash /næS/ } \\
\text { gnome/nəひm/ }\end{array}$ & $\begin{array}{l}\text { gnaw /nว:/ } \\
\text { sign /sain/ }\end{array}$ & $\begin{array}{l}\text { gnat /næt/ } \\
\text { hang /hæn/ }\end{array}$ \\
\hline 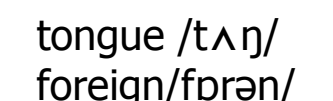 & $\begin{array}{l}\text { reign /reIn/ } \\
\text { resian /rIzaIn/ }\end{array}$ & $\begin{array}{l}\text { thing / ӨIn/ } \\
\text { desian /dIzaIn/ }\end{array}$ \\
\hline $\begin{array}{l}\text { young /y^๖/ } \\
\text { fling /fIIn/ } \\
\text { arraign / ərein/ } \\
\text { campaign /kæm }\end{array}$ & $\begin{array}{l}\text { strong /strpy/ } \\
\text { song /spy/ } \\
\text { monsignor/monsI } \\
\text { eIn/ }\end{array}$ & $\begin{array}{l}\text { sing /sin/ } \\
\text { sovereign /sDvrin/ } \\
\text { ijə/ }\end{array}$ \\
\hline
\end{tabular}

\section{Silent H}

$\begin{array}{lll}\text { hour/auə/ } & \text { honest / DnIst/ } & \text { honour / Dnə/ } \\ \text { heir/eə/ } & \text { exhaust /Igzว:st/ } & \text { exhibit }\end{array}$

handsome /hænsəm/

Wednesday/wenzdeI/ 
vehicle /vi:kl/ shepherd/sepз:d/ ghost /gəひst/

rhyme /raIm/ which /wit /

rheumatism /ru:mətizəm/

\section{Silent K}

\begin{tabular}{|c|c|c|}
\hline $\begin{array}{l}\text { Know /nəひ/ } \\
\text { Knight /nait/ } \\
\text { Kneel /nI:I/ } \\
\text { knot /nnt/ }\end{array}$ & $\begin{array}{l}\text { knit /nIt/ } \\
\text { knee/nI:/ } \\
\text { knew /nju:/ } \\
\text { knoll /nəəl/ }\end{array}$ & $\begin{array}{l}\text { knock /nvk/ } \\
\text { knife/naIf/ } \\
\text { knob/nob/ } \\
\text { knead /nI:d/ }\end{array}$ \\
\hline $\begin{array}{l}\text { Knickers /nIkəz/ } \\
\text { knowledge/nolid3 }\end{array}$ & $\begin{array}{l}\text { knave /neIv/ } \\
\text { Knapsack /na }\end{array}$ & knuckle /n^kl/ \\
\hline
\end{tabular}

\section{Silent $\mathbf{L}$}

Could /kud/ or /kəd/ would /wod/ or /wəd/

Should / $\mathrm{sud} /$ or / $\mathrm{J} \partial \mathrm{d} / \quad$ calf /ka:f/ chalk / $\mathrm{t}$ j:k/

Palm /pa:m/

half /ha:f/

psalm/sa:m/

salmon /sæmən/ halves /hævs/

\section{Silent M} walk/wo:k/ yolk /jəək/ behalf /biha:f/

Mnemonics /nəmbniks/

\section{Silent N}

$\begin{array}{lll}\text { hymn } / \mathrm{hIm} / & \text { solemn /sbləm/ } & \text { condemn /kəndem/ } \\ \text { column /kbləm/ } & \text { autumn /ว:t əm/ } & \text { damn /dæm/ }\end{array}$

\section{Silent P}

Psalm /sa:m/ psychology /saIkblədzI/ psychic/saIkIk/

Pseudo /sju:dər/ corps/ko:/ empty /emtI/

Cupboard /kəbə:d/ Sampson /sæmsn/ receipt/rIsI:t/

Coup /ku:/

pseudonym /sju:dənIm/

psychiatry /saIkaIətri/

pneumonia /nju:məənIə/

\section{Silent S}

Island/aIlond/ isle /aIl/ 


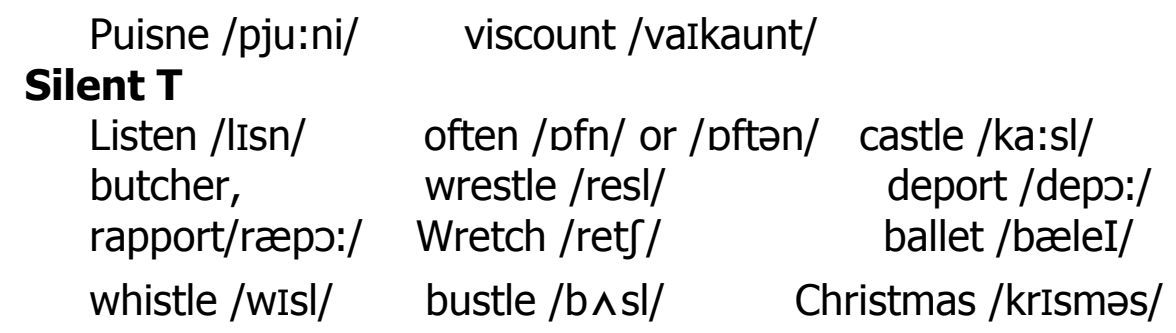

\section{Silent $\mathbf{U}$}

Guess /ges/ quest /kwest/ guard/ga:d/

Guide /gaid/ rouge /rəorg/ colleague /kbli:g/

Guitar /gita:/ guinea /gInI/ vague /veIg/

\section{Silent $\mathbf{W}$}

guild /gild/ guilt /gilt/

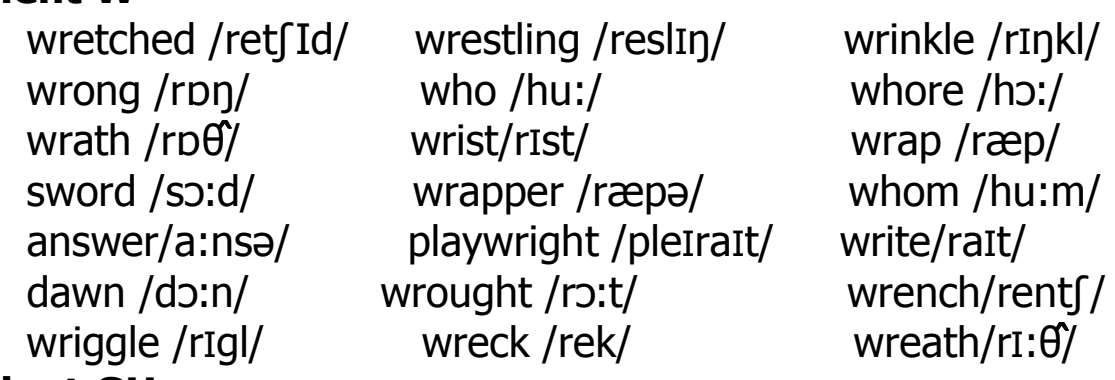

\section{Silent GH}

\begin{tabular}{|c|c|c|}
\hline $\begin{array}{l}\text { blight/blaIt/ } \\
\text { caught /kJ:t/ } \\
\text { straight /streIt/ } \\
\text { fight /faIt/ } \\
\text { height /haIt/ } \\
\text { thigh / ӨaI/ }\end{array}$ & $\begin{array}{l}\text { bright /brait/ } \\
\text { delight /dilaIt/ } \\
\text { sigh /saI/ } \\
\text { light /lait/ } \\
\text { naught /no:t/ } \\
\text { weight / weit/ }\end{array}$ & $\begin{array}{l}\text { bought /bo:t/ } \\
\text { might /mait/ } \\
\text { neighbour / neIbə/ } \\
\text { right /rait/ } \\
\text { taught /to:t/ } \\
\text { plough /plau/ }\end{array}$ \\
\hline
\end{tabular}

\section{Silent UE}

Catalogue /kætəlng/ dialogue /daiəlng/

Epilogue /epilpg/ prologue /prəzlng/

\section{Suggestions/Recommendation}

Since every nation is striving for recognition in the globalization project, no one can afford to be left behind, the 
curriculum planners need not live in the delusion of native or local technology as some people are campaigning. They should inculcate the teaching of English language into the curriculum from the kindergarten level in order to create relevance and competitive edge for the average Ethiopian in the world system

As language teachers, we should Endeavour to teach the language and not about the language. A language is what the native speakers say not what some people think they ought to say. It is therefore necessary to teach pronunciation and pronunciation problems to students from the early stage of education.

Let the students know aspects of their pronunciation that result in other people being unable to understand them. They should also be given opportunity to practice aspects of the English sound system which are crucial for their own improvement. This could be done through minimal pairs, dialogues and other conversation practices.

Finally, the attempt is not to completely eradicate a foreign accent but the goal of the teacher is to bring an improvement in their pronunciation especially as regards critical error responsible for incomprehensibility. 


\section{References}

Brown, Gillian. (1990) Listening to Spoken English. 2nd Ed. Essex- England: Longman Group Ltd.

Gimson, A.C. (1980) An Introduction to the Pronunciation of English.3rd Ed.London: dward Arnold (Publishers) Ltd.

Finocchiara, Mary. (1989) English as a Second/Foreign Language. 4th Ed. New Jersey: Prentice Hall Inc.

Hornby, A.S.(2005) Oxford Advanced Learner's Dictionary of Current English. 7th Ed. New York: Oxford University Press.

Jones, Daniel. (1995) English Pronouncing Dictionary. 14th Revised by A.C. Gimson. New Delhi: Universal Book Stall.

Kuiper, Koenraad and W. Scott Allan.(1996) An Introduction to English Language -Sound, Word and Sentence. London: Macmillan Press Ltd.

Ladefoged, Peter. (1975) A Course in Phonetics. USA: Harcourt Brace Jovanovich Inc.

Roach, Peter.(1983) English Phonetics and Phonology. Cambridge: Cambridge University Press.

Sethi, J. and P.V. Dhamija.(2000) A Course in Phonetics and Spoken English. 2nd Ed.New Delhi: Prentice- Hall.

Seyoun, T.(1996): Attempt at Educational Reforms in Ethiopia. Ethiopian Journal of Education Vol. 9 no. 1

Tench, Paul. (1981) Pronunciation Skills. London: Macmillan Publishers Ltd.

Umera-Okeke, N. (Unpublished) English Pronunciation Basics. 